Gut and Liver, Vol. 12, No. 6, November 2018, pp. 682-693

\title{
Effects of $17 \beta$-Estradiol on Colonic Permeability and Inflammation in an Azoxymethane/Dextran Sulfate Sodium-Induced Colitis Mouse Model
}

\author{
Chin-Hee Song ${ }^{1}$, Nayoung Kim ${ }^{1,2}$, Sung Hwa Sohn ${ }^{1}$, Sun Min Lee ${ }^{1}$, Ryoung Hee Nam ${ }^{1}$, Hee Young Na ${ }^{4}$, Dong Ho Lee ${ }^{1,2}$, and \\ Young-Joon Surh ${ }^{3}$ \\ ${ }^{1}$ Department of Internal Medicine, Seoul National University Bundang Hospital, Seongnam, ${ }^{2}$ Department of Internal Medicine and Liver \\ Research Institute, Seoul National University College of Medicine, ${ }^{3}$ Tumor Microenvironment Global Core Research Center, Seoul National \\ University College of Pharmacy, Seoul, and ${ }^{4}$ Department of Pathology, Seoul National University Bundang Hospital, Seongnam, Korea
}

Background/Aims: Intestinal barrier dysfunction is a hallmark of inflammatory bowel diseases (IBDs) such as ulcerative colitis. This dysfunction is caused by increased permeability and the loss of tight junctions in intestinal epithelial cells. The aim of this study was to investigate whether estradiol treatment reduces colonic permeability, tight junction disruption, and inflammation in an azoxymethane (AOM)/dextran sodium sulfate (DSS) colon cancer mouse model. Methods: The effects of $17 \beta$-estradiol (E2) were evaluated in ICR male mice 4 weeks after AOM/DSS treatment. Histological damage was scored by hematoxylin and eosin staining and the levels of the colonic mucosal cytokine myeloperoxidase (MPO) were assessed by enzyme-linked immunosorbent assay (ELISA). To evaluate the effects of E2 on intestinal permeability, tight junctions, and inflammation, we performed quantitative real-time polymerase chain reaction and Western blot analysis. Furthermore, the expression levels of mucin 2 (MUC2) and mucin 4 (MUC4) were measured as target genes for intestinal permeability, whereas zonula occludens 1 (ZO-1), occludin (OCLN), and claudin 4 (CLDN4) served as target genes for the tight junctions. Results: The colitis-mediated induced damage score and MPO activity were reduced by E2 treatment $(p<0.05)$. In addition, the mRNA expression levels of intestinal barrier-related molecules (i.e., MUC2, ZO-1, OCLN, and CLDN4) were decreased by AOM/DSS-treatment; furthermore, this inhibition was rescued by E2 supplementation. The mRNA and protein expression of inflammation-related genes (i.e., KLF4, NF-кB, iNOS, and COX-2) was increased by AOM/DSS-treatment and ameliorated by E2. Conclusions: E2 acts through the estrogen receptor $\beta$ signaling pathway to elicit anti-inflammatory effects on intestinal barrier by inducing the expression of MUC2 and tight junction molecules and inhibiting pro-inflammatory cytokines. (Gut Liver 2018;12:682-693)

Key Words: AOM/DSS colitis mouse model; Permeability; Tight junction; Inflammation; Estrogen

\section{INTRODUCTION}

Colorectal cancer (CRC) is the third leading cause of cancer deaths in both men and women in the United States, with an estimated 97,220 new cancer cases and 50,630 cancer deaths in $2018^{1}$ and the major cause of death in women with age above 65 years old in Korea. ${ }^{2}$ When CRC occurs in the patients with inflammatory bowel diseases (IBD) including ulcerative colitis and Crohn's disease, which are a chronic disorder of the intestine, ${ }^{3}$ their progression to CRC is strongly associated with extent of inflammation and duration of IBD. Chronic inflammatory status drive cells to malignant transformation to promote tumorigenesis by oxidative stress and the pro-inflammatory factors such as interleukin 6 (IL-6), tumor necrosis factor- $\alpha$ (TNF- $\alpha$ ) and myeloperoxidase (MP0).,

The most widely used animal models in the investigation of the molecular pathogenesis in colitis and colitis-associated CRC is a mouse model with azoxymethane (AOM) and dextran sulfate sodium (DSS) ${ }^{6-8}$ mainly because it is a powerful, reproducible, and cost-effective model that utilize toxic chemical induction of oxidative DNA damage. ${ }^{9-11}$ Furthermore, this model is simulates the multistep tumor progression based on the aberrant crypt foci-adenoma-carcinoma sequence with the molecular alterations assessed in specific phases of the carcinogenic process. $^{10}$

Intestinal barrier dysfunction is a hallmark IBD and it is

Correspondence to: Nayoung Kim

Department of Internal Medicine, Seoul National University Bundang Hospital, 82 Gumi-ro 173beon-gil, Bundang-gu, Seongnam 13620, Korea

Tel: +82-31-787-7008, Fax: +82-31-787-4051, E-mail: nayoungkim49@empas.com

Received on May 16, 2018. Revised on August 28, 2018. Accepted on September 12, 2018. Published online October 29, 2018

pISSN 1976-2283 eISSN 2005-1212 https://doi.org/10.5009/gnl18221

@. This is an Open Access article distributed under the terms of the Creative Commons Attribution Non-Commercial License (http://creativecommons.org/licenses/by-nc/4.0) which permits unrestricted non-commercial use, distribution, and reproduction in any medium, provided the original work is properly cited. 
caused by increased permeability and loss of tight junctions (TJs) in intestinal epithelial cells. The intestinal barrier is mainly composed of the mucus and the epithelial TJs which are proposed as potential markers of intestinal permeability. ${ }^{12-16}$ It is regulated by multiple factors such as the intestinal microbiota, ${ }^{17}$ food, ${ }^{18}$ cytokines and immune cells. ${ }^{19}$ At this point, the intestinal epithelium plays a key role to prevent permeation regulated by intestinal permeability modulating factors. In addition, the most important barrier is composed of colonic mucin, which is secreted from the colonic mucosa. The major component of the colonic mucus layer is the gel-forming mucin 2 (MUC2) and MUC2 knockout mice spontaneously developed colitis and CRC. ${ }^{20,21}$ In contrast, MUC4 knockout mice were found to be highly resistant to chemical-induced colitis and CRC. ${ }^{22}$ Another epithelial TJ molecules act as key multi-protein complexes to maintain the intestinal barrier while regulating permeability of nutrients and water. ${ }^{23}$ There are many evidences that the expressions of mucin and TJ molecules become different in the CRC in comparison to normal conditions. That is, the expression of Zonula occludens-1 (ZO-1) and occludin (OCLN) was downregulated in CRC, but the expressions of claudin (CLDN) 1, 2, 3, and 4 were up-regulated in CRC. ${ }^{24}$ In addition, an over expression of protease-activated receptor 2 (PAR-2), which is a family member of $\mathrm{G}$ protein-coupled receptors, associated with the increased mucosal permeability in a guinea pig model of postoperative ileus $^{25}$ and reflux esophagitis in human. ${ }^{26}$

Epidemiological, clinical and experimental studies reported that $17 \beta$-estradiol (E2), the predominant form of estrogen, confers protection against colon cell proliferation and malignant transformation, reducing the incidence of adenoma and carcinoma in colon. ${ }^{27,28}$ The physiological effects of estrogens are mediated by its receptors which are estrogen receptor $\alpha(E R \alpha)$ and $\beta$ (ER $\beta$ ) encoded by different genes, and they have different expression patterns in colon. ER $\alpha$ was minimally expressed in colon cancer cells, and ER $\beta$ expression was significantly lower in colon cancer cells than in normal colonic epithelium. ${ }^{27}$ In addition, ER $\beta$-selective agonists have been proposed to be therapeutically effective in the model of IBD and arthritis. ${ }^{29}$

Our research team have previously reported that colitisassociated colon carcinogenesis in AOM/DSS murine model was induced more severely in male mice than female by way of the inflammatory mediators such as IL- $1 \beta$ and MPO ${ }^{30}$ Furthermore, we found that E2 $(10 \mathrm{mg} / \mathrm{kg})$, the major female sex hormone, inhibited the initiation of CRC by up-regulating nuclear factor erythroid 2-related factor 2, a transcriptional factor-related pathways in the AOM/DSS-treated male ICR mice. ${ }^{28}$

From this background, we hypothesized that estrogen inhibits AOM/DSS-colitis induced intestinal permeability and inflammation. To evaluate this hypothesis, the aim of this study was to investigate the levels of colonic mucosal cytokine and the gene expressions related to permeability, TJs, and inflammation in male AOM/DSS with or without E2, and to compare these markers between male and female mice.

\section{MATERIALS AND METHODS}

\section{Animals}

Four-week-old male and female ICR mice (Orient Co., Ltd., Seoul, Korea) were housed in cages and maintained at $23^{\circ} \mathrm{C}$ with a 12/12-hour light/dark cycle under specific pathogen-free conditions. All animal experimental procedures were approved by the Institutional Animal Care and Use Committee (IACUC) of Seoul National University Bundang Hospital (BA1310-139/09101). The procedures were in accordance with the ARRIVE (Animals in Research: Reporting In Vivo Experiments) statement.

\section{Experimental design}

The experimental design is presented in Fig. 1A. After 1 week of acclimatization, male and female mice were randomized into five groups. Group 1 comprised male control mice (M_Con.) $(\mathrm{n}=4)$. Group 2 comprised male mice treated with AOM/DSS (M_ AOM/DSS) ( $n=6)$. Group 3 comprised AOM/DSS-treated male mice administered estradiol (M_AOM/DSS/E2) (n=6). Group 4 comprised female control mice (F_Con.) ( $n=4)$. Group 5 comprised AOM/DSS-treated female mice (F_AOM/DSS) $(n=6)$. AOM/DSS-treated male and female mice were intraperitoneally injected with AOM (10 mg/kg; Sigma-Aldrich, St. Louis, MO, USA) on day 0 in the experimental schedule. For induction of colitis, 2.5\% (w/v) DSS (MP Biomedicals, Aurora, OH, USA) was supplied in drinking water for 7 days 1 week following the injection of $\mathrm{AOM}^{31}$. The quantity of DSS consumed in the drinking water was checked on day 7, 9, and 11. M_AOM/DSS/E2 mice were intraperitoneally injected each day for 7 days with E2 (10 mg/kg; Sigma-Aldrich, E8876) dissolved in olive oil. The injections were done during the same period of DSS consumption. Animals were euthanized by $\mathrm{CO}_{2}$ asphyxiation at week 4 after AOM injection (Fig. 1A).

\section{Histology and scoring of microscopic damage}

The proximal colon up to $1.5 \mathrm{~cm}$ from the ileocecal valve, the rectum up to $1.5 \mathrm{~cm}$ from the anal verge, and the colonic segments containing any grossly proven polyps were fixed with phosphate-buffered formalin and embedded in paraffin. The $5-\mathrm{mm}$ sections were stained with hematoxylin and eosin. Histological severity was assessed using microscopic damage score reflecting colonic epithelial damage and depth of infiltration with inflammatory cells as previously described. ${ }^{32}$ It was evaluated by two researchers in a blinded manner.

Immunohistochemical (IHC) analysis of ZO-1 was performed. Tissue sections were treated with 3\% hydrogen peroxide and nonspecific binding sites were blocked. The sections were incubated with anti-ZO-1 antibodies (61-7300, Thermo Fisher Scientific, Waltham, MA, USA). An automatic immunostainer (BenchMark XT; Ventana Medical Systems, Inc, Tucson, AZ, 
A

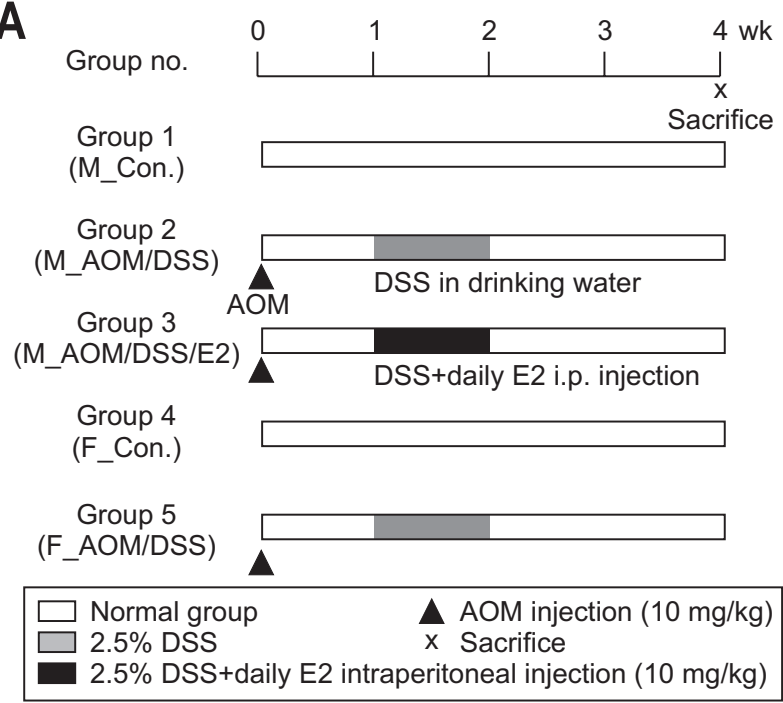

C

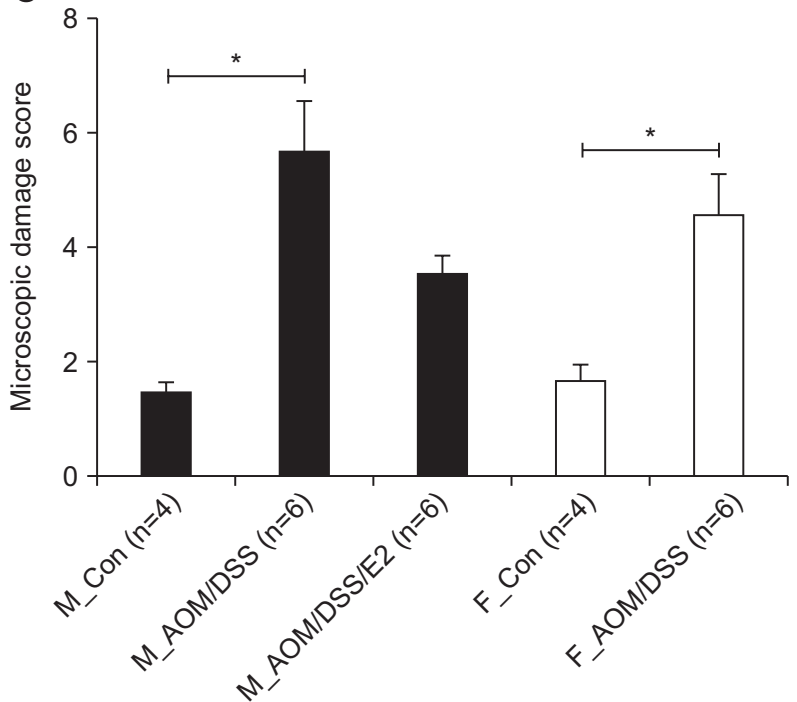

B
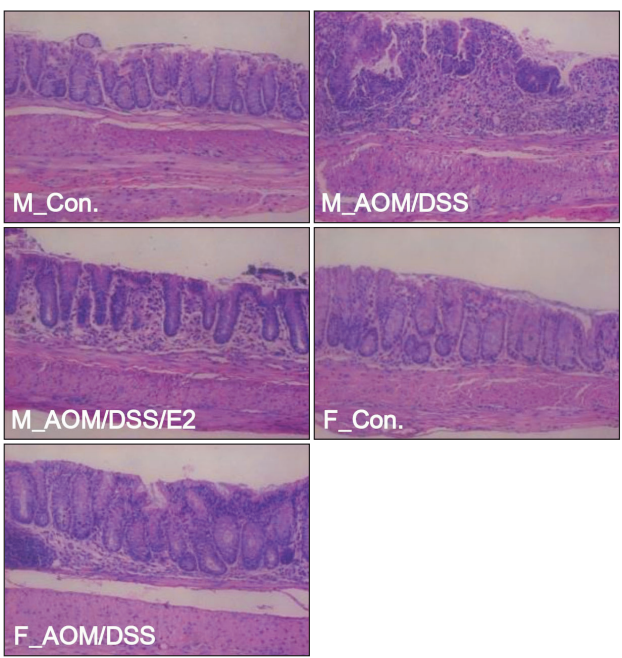

D

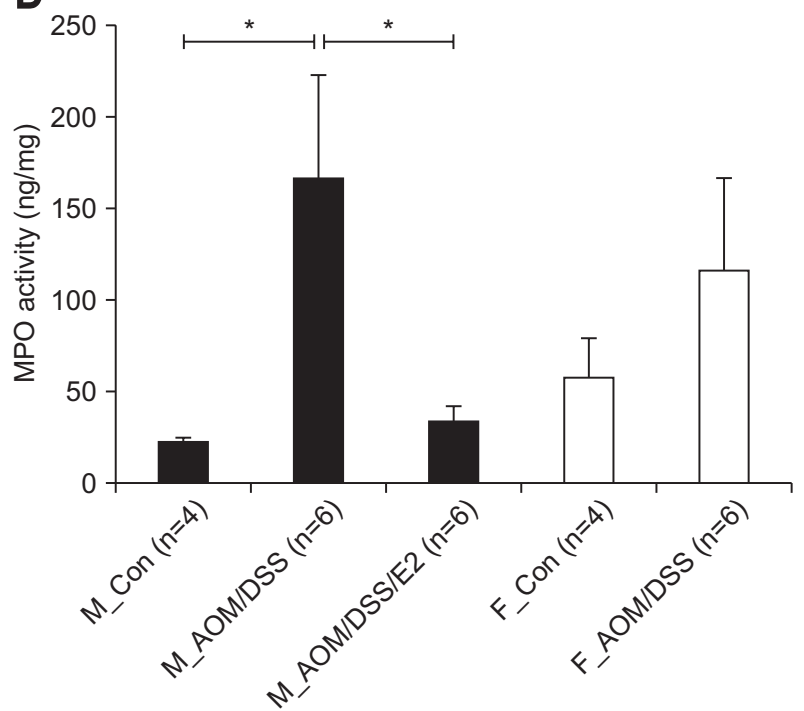

Fig. 1. Inhibitory effects of 17ß-estradiol (E2) on the inflammation index in azoxymethane/dextran sulfate sodium (AOM/DSS)-induced colitis in mice. (A) Experimental protocol for evaluating the effects of E2 on AOM/DSS-induced colitis in mice. The mice were injected with AOM on day 0, and 2.5\% DSS in drinking water and estradiol supplements were provided from days 8 to 14. Mice were sacrificed on week 4 after AOM injection. (B) Inhibition of AOM/DSS-induced inflammatory cell infiltration by E2. Representative hematoxylin and eosin staining images of distal colonic mucosa (magnification, $\times 100$ ). (C) The AOM/DSS-induced microscopic damage score was reduced by E2. (D) A0M/DSS-induced myeloperoxidase (MPO) activity was inhibited by E2.

M_Con., male control mice; M_AOM/DSS, male mice treated with AOM/DSS; M_AOM/DSS/E2, A0M/DSS-treated male mice administered estradiol; F_Con., female control mice; F_AOM/DSS, female mice treated with AOM/DSS. ${ }^{*} \mathrm{p}<0.05$ for comparison between two groups.

USA) and UltraView Universal DAB detection kit (Ventana Medical Systems) were used for immunostaining. The expression percentage was assessed as the ratio of the number of the cells stained with ZO-1 to the total cells counted per section. Z0-1 expression was quantified using ImageJ 1.50i software, with the Cell Counter plugin. The reported values are the average of different images from different mice within each group.

\section{Measurement of inflammatory cytokine}

The levels of MPO activity in the colonic tissues were measured using a mouse MPO enzyme-linked immunosorbent assay kit (Hycult Biotechnology, The Netherlands), according to the manufacturer's instruction. Every assay was performed in triplicate.

\section{Quantitative real-time PCR}

Total RNA was prepared from colon tissue using Trizol reagent (Invitrogen, Carlsbad, CA, USA) according to the manufacturer's instruments, and quantified using a NanoDrop ND-1000 device (Thermo Scientific, Wilmington, DE, USA). Complementary DNA (cDNA) was synthesized using the High Capacity cDNA reverse Transcription Kit (Applied Biosystems, 
Table 1. List of Oligonucleotides for qRT-PCR

\begin{tabular}{|c|c|}
\hline Gene & Sequence $\left(5^{\prime} \rightarrow 3^{\prime}\right)$ \\
\hline \multirow[t]{2}{*}{ MUC2 } & F: TTT CAA GCA CCC CTG TAA CC \\
\hline & R: AGG TCC TGG TGT TGA ACC TG \\
\hline \multirow[t]{2}{*}{ MUC4 } & F: CCC CCA TCT TTC TGT CTC AA \\
\hline & R: AGG ATG GAA TTG GTG TT TG \\
\hline \multirow[t]{2}{*}{ ZO-1 } & F: ACT ATG ACC ATC GCC TAC GG \\
\hline & R: GGG GAT GCT GAT TCT CAA AA \\
\hline \multirow[t]{2}{*}{ OCLN } & F: CGG TAC AGC AGC AAT GGT AA \\
\hline & R: CTC CCC ACC TGT CGT GTA GT \\
\hline \multirow[t]{2}{*}{ CLDN4 } & F: GGG GAT CAT CCT GAG TTG TG \\
\hline & R: CAC TGC ATC TGA CCT GTG CT \\
\hline \multirow[t]{2}{*}{ KLF4 } & F: CCA AAG AGG GGA AGA AGG TC \\
\hline & R: CTG TGT GAG TTC GCA GGT GT \\
\hline \multirow[t]{2}{*}{ TNF- $\alpha$} & F: ACG GCA TGG ATC TCA AAG AC \\
\hline & R: GTG GGT GAG GAG CAC GTA GT \\
\hline \multirow[t]{2}{*}{ IL-6 } & F: CTG CAA GAG ACT TCC ATC CAG TT \\
\hline & R: GAA GTA GGG AAG GCC GTG G \\
\hline \multirow[t]{2}{*}{ IL-1 $\beta$} & F: CAG GCA GGC AGT ATC ACT CA \\
\hline & R: TGT CCT CAT CCT GGA AGG TC \\
\hline \multirow[t]{2}{*}{$\mathrm{ER} \alpha$} & F: TGG GCT TAT TGA CCA ACC TAG CA \\
\hline & R: AGA ATC TCC AGC CAG GCA CAC \\
\hline \multirow[t]{2}{*}{ ER $\beta$} & F: TCT TTG CTC CAG ACC TCG TT \\
\hline & R: AAT CAT GGC CTT CAC ACA CA \\
\hline \multirow[t]{2}{*}{ GAPDH } & F: TTC ACC ACC ATG GAG AAG GC \\
\hline & R: GGC ATG GAC TGT GGT CAT GA \\
\hline
\end{tabular}

qRT-PCR, quantitative real-time polymerase chain reaction; MUC2, mucin 2; MUC4, mucin 4; ZO-1, zonula occludens 1; OCLN, occludin; CLDN4, claudin 4; KLF4, Krüppel-like factor 4; TNF- $\alpha$, tumor necrosis factor- $\alpha$; IL, interleukin; ER, estrogen receptor; GAPDH, glyceraldehyde-3-phosphate dehydrogenase.

Foster City, CA, USA). The cDNA was used to perform quantitative real-time polymerase chain reaction (qRT-PCR) using specific primers (listed in Table 1) and Power SYBR Green PCR Master mix (Thermo Fisher Scientific) in Viia7 instrument (Applied Biosystems). Expression levels of the genes were normalized to that of GAPDH.

\section{Western blot analysis}

Total protein was extracted from colon tissue using RIPA buffer (Cell Signaling Technology, Beverly, MA, USA) containing protease and phosphatase inhibitors. Cytoplasmic and nuclear lysates were separated using a NE-PER Nuclear Cytoplasmic Extraction Reagent kit (Pierce, Rockford, IL, USA) according to the manufacturer's instructions. Protein concentration was determined using the BCA protein assay reagent (Pierce). Protein samples were mixed with an equal volume of $5 \times$ sodium dodecyl sulfate (SDS) sample buffer, boiled for 5 minutes, and then separated using $8 \%$ to 15\% SDS-PAGE. After electrophoresis,
Table 2. List of Antibodies and Its Characteristics

\begin{tabular}{lll}
\multicolumn{1}{c}{ Antigen } & \multicolumn{1}{c}{ Antibody } & \multicolumn{1}{c}{ Dilution } \\
\hline NF-кB P65 & Santa Cruz Biotechnology (sc8008) & WB $(1: 1,000)$ \\
iNOS & BD Biosciences (\#610328) & WB $(1: 500)$ \\
COX-2 & Santa Cruz Biotechnology (sc1745) & WB $(1: 1,000)$ \\
Z0-1 & Thermo Fisher Scientific (\#61-7300) & IHC $(1: 100)$ \\
$\beta$-Actin & Santa Cruz Biotechnology (sc47778) & WB $(1: 1,000)$ \\
Lamin B & Santa Cruz Biotechnology (sc6216) & WB $(1: 1,000)$ \\
\hline
\end{tabular}

NF- $\mathrm{B}$ P65, nuclear factor $\kappa \mathrm{B}$ P65; WB, Western blot; iNOS, inducible nitric oxide synthase; COX-2, cyclooxygenase-2; IHC, immunohistochemical; ZO-1, zonula occludens 1.

proteins were transferred to polyvinylidene difluoride (PVDF) membranes. The membranes were blocked with 5\% nonfat dry milk in tris-buffered saline with $0.1 \%$ Tween-20 buffer (TBST) for 1 hour at room temperature. Membranes were incubated overnight at $4^{\circ} \mathrm{C}$ with specific antibodies. Primary antibodies were removed by washing the membranes three times in TBS$\mathrm{T}$, and incubated for 2 hours with horseradish peroxidase-conjugated anti-rabbit, anti-goat or anti-mouse immunoglobulin (Santa Cruz Biotechnology, Dallas, TX, USA). Following three washes with TBS-T, the antigen-antibody complexes were detected with the ECL Prime Western Blotting Detection Kit (GE Healthcare Biosciences, Piscataway, NJ, USA). The intensity of the blots was quantified by densitometry analysis using ImageJ software (National Institutes of Health, Bethesda, MD, USA). The antibodies are listed in detail in the Table 2.

\section{Statistical analysis}

Data are expressed as mean \pm standard error of the mean. Statistical significance was examined with Mann-Whitney test or Fisher exact test. A p-value $<0.05$ was considered to indicate a statistical significance. All statistical analyses were conducted using GraphPad Prism software (GraphPad, La Jolla, CA, USA) and SPSS version 18.0 (SPSS Inc., Chicago, IL, USA).

\section{RESULTS}

\section{E2 ameliorates histologic evidence of colonic inflammation}

We first analyzed severity of colitis to evaluate early impacts of estradiol. Representative histologic photos (Fig. 1B) and microscopic damage score (Fig. 1C) showed that the AOM/DSStreated male mice with E2 administration had significantly less infiltration of inflammatory cells and mild cryptic damage compared to the AOM/DSS-treated male mice on week 4 ( $p=0.054$ for microscopic damage score) (Fig. 1C). The AOM/DSS-treated male mice with E2 administration showed significantly lower level of MP0, a mediator associated with intestinal inflammation, compared to the AOM/DSS-treated male mice ( $\mathrm{p}=0.015$ for MPO activity) (Fig. 1D). Taken together, our findings suggest that estrogen reduces the severity of colitis. 

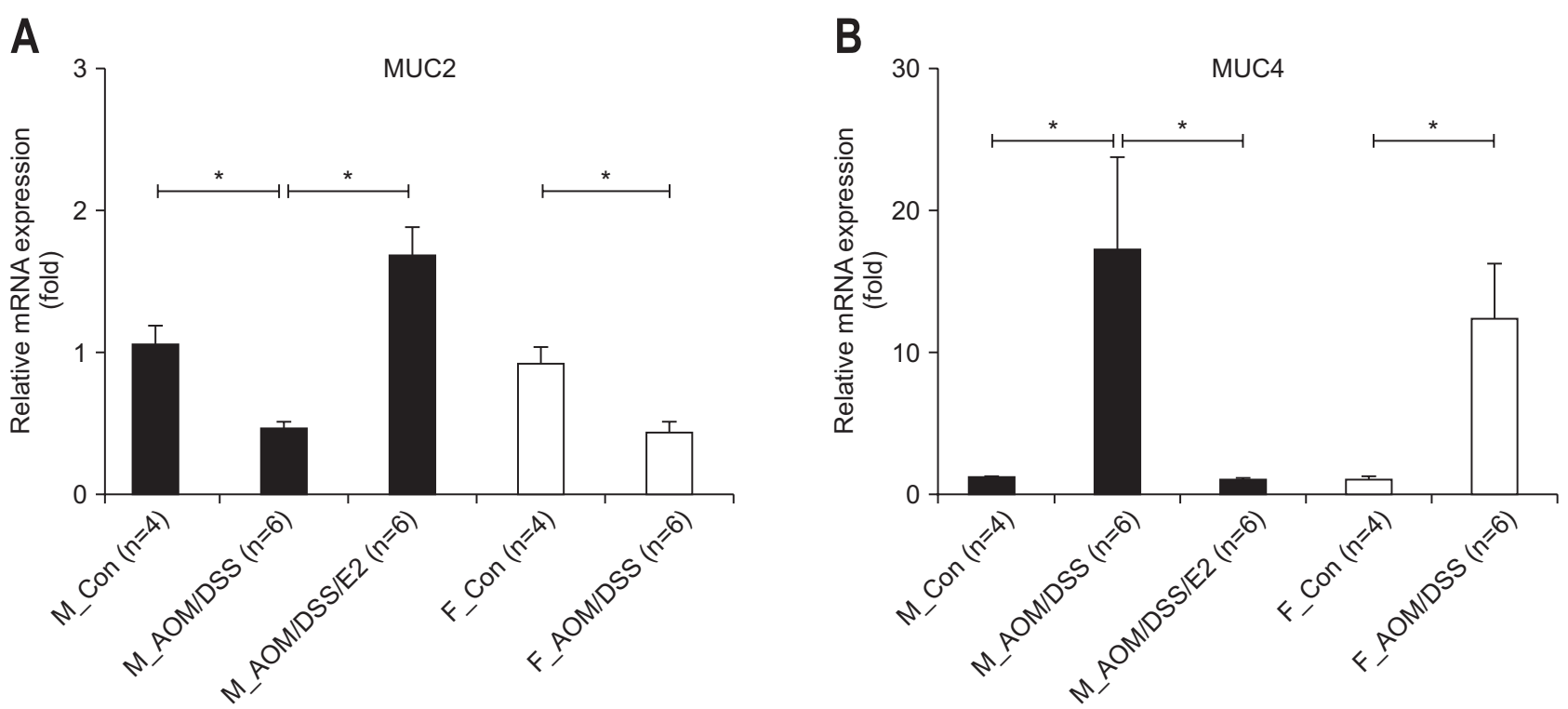

Fig. 2. Differentially regulated expression of the mucin gene by $17 \beta$-estradiol (E2) supplementation in azoxymethane/dextran sulfate sodium (AOM/DSS)-induced colitis in mice. (A) AOM/DSS-mediated reductions in mucin 2 (MUC2) mRNA expression were enhanced by E2. (B) A0M/ DSS-induced expression of MUC4 mRNA was inhibited by E2. Expression of MUC2 and MUC4 in the colonic mucosa was measured by qRT-PCR analysis. GAPDH was used as an internal control to normalize the expression.

qRT-PCR, quantitative real-time polymerase chain reaction; GAPDH, glyceraldehyde-3-phosphate dehydrogenase; M_Con., male control mice; M_ AOM/DSS, male mice treated with AOM/DSS; M_AOM/DSS/E2, AOM/DSS-treated male mice administered estradiol; F_Con., female control mice; F_AOM/DSS, female mice treated with AOM/DSS. *p<0.05 for comparison between two groups.

\section{E2 differently regulates the expression of mucin genes, MUC2 and MUC4}

Since MUC2 is the major secreted mucin within the goblet cells in both the small and large intestine and it acts as the fundamental component of the mucus barrier. ${ }^{33}$ MUC4 is one of the transmembrane mucins have a single membrane spanning region. To investigate the effect of E2 on the mucus layer as a first barrier on the intestinal protection, we analyzed the mRNA expression level of mucin genes (i.e., MUC2 and MUC4) in mouse colon tissue sample. As shown in Fig. 2A, the inhibited expression of MUC2 in AOM/DSS-treated male mice was recovered by E2 supplementation ( $\mathrm{p}<0.001)$. In female, MUC2 mRNA expression was significantly diminished by AOM/DSS treatment compare to control ( $\mathrm{p}=0.001$ ) (Fig. 2A). In contrast to MUC2 gene expression regulation, in both male and female, MUC4 mRNA expression was enhanced by AOM/DSS treatment. In addition, the AOM/DSS-mediated enhanced expression of MUC4 was inhibited by E2 administration in male mice $(p<0.001)$ (Fig. 2B). There was no difference between male and female in both genes. These results suggest that E2 promotes intestinal barrier function through regulation of mucin gene expression act as a first intestinal barrier.

\section{E2-mediated up-regulation of TJ molecules}

Next, to figure out the additional effect of E2 on intestinal permeability, we assessed TJ molecules mRNA expression by qRT-PCR analysis. Among TJ molecules, ZO-1 mRNA expres- sion was approximately 1.7-fold decreased by AOM/DSS treatment compare to control in male. And, the decreased expression was altered to basal level by E2 administration in AOM/DSStreated male mice ( $\mathrm{p}=0.001)$ (Fig. $3 \mathrm{~A})$. In addition, the mRNA expressions of OCLN and CLDN4 were approximately 2.6-fold $(\mathrm{p}<0.001)$ (Fig. 3B) and 4-fold ( $\mathrm{p}=0.005)$ (Fig. 3C) diminished by AOM/DSS treatment, respectively. The down-regulated expressions were significantly recovered to basal level by E2 supplementation in AOM/DSS-treated male mice $(\mathrm{p}=0.018$ for OCLN, $\mathrm{p}=0.004$ for CLDN4). In contrast to male, the mRNA expressions of TJ molecules such as ZO-1, OCLN, and CLDN4 were strongly up-regulated by AOM/DSS treatment compare to control in female group (Fig. 3A-C). Consistent with mRNA expression, the percentage of $\mathrm{ZO}$-1-positive cells stained into the plasma membrane was significantly repressed by AOM/DSS treatment compare to control in male $(\mathrm{p}<0.001)$ (Fig. 3D). And, the decreased expression was recovered by E2 supply in AOM/DSStreated male mice $(\mathrm{p}=0.026)$ (Fig. 3D). In contrast to male, ZO1-positive cells were approximately $2.5 \%$ increased by AOM/ DSS treatment compare to control in female group, but not significantly (Fig. 3D). These data suggest that exogenous estrogen has protective role on the colitis-mediated intestinal permeability through up-regulation of TJ-related gene expression.

\section{Suppression of NF- $\kappa$ B-dependent signaling pathway by E2 supplementation}

To further evaluate estrogen effects on inflammatory factors in molecular levels, we measured nuclear factor $\kappa \mathrm{B}(\mathrm{NF}-\kappa \mathrm{B})$ - 

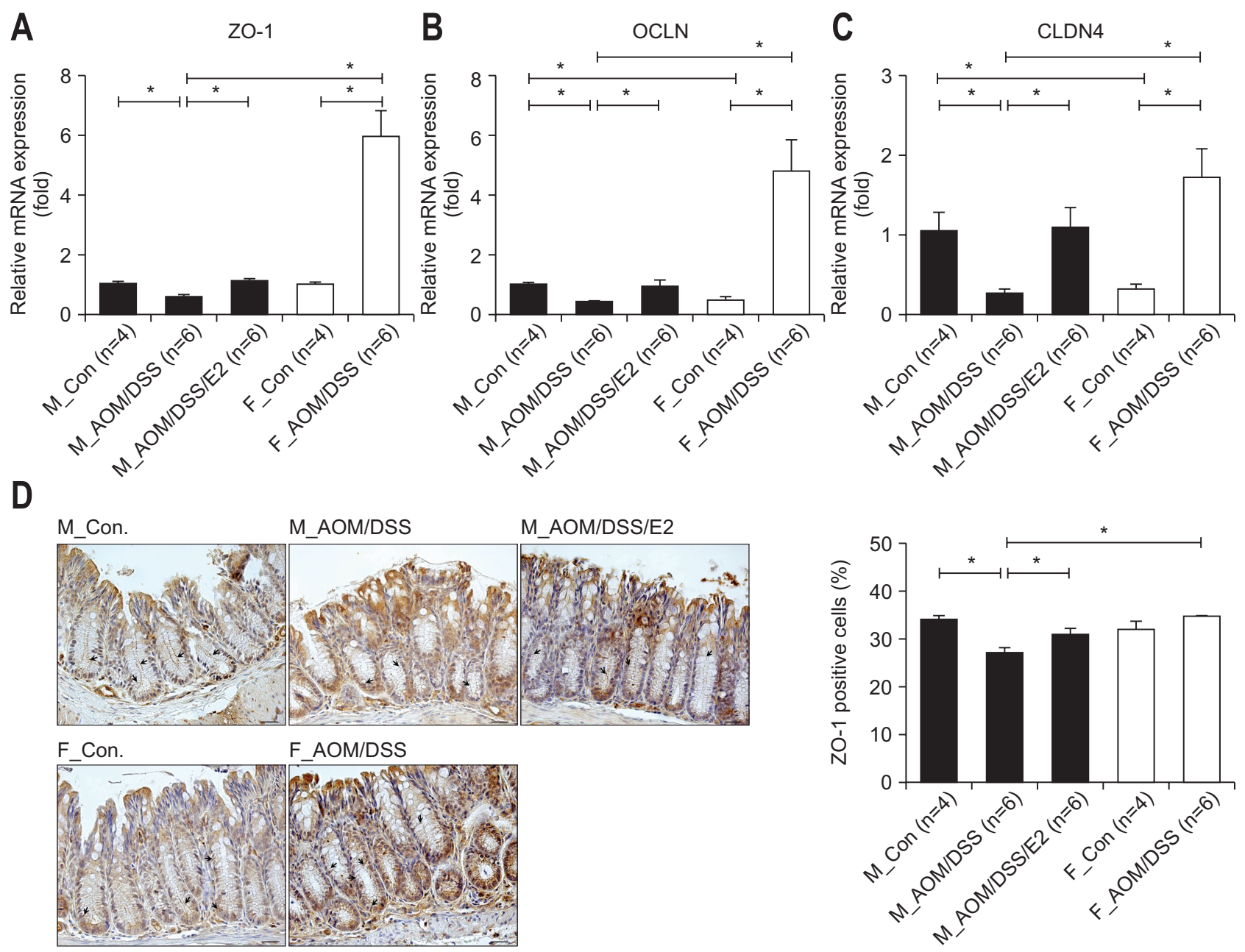

Fig. 3. Effects of $17 \beta$-estradiol (E2) and sex-associated differences on the expression of tight junction molecules in azoxymethane/dextran sulfate sodium (AOM/DSS)-induced colitis in mice. (A-C) In the male group, AOM/DSS-mediated suppression of ZO-1 (A), OCLN (B), and CLDN4 (C) mRNA expression was recovered by E2 treatment. In females, the expression levels of these genes were increased after AOM/DSS treatment. GAPDH expression was used as an internal control to normalize the expression. (D) Immunohistochemical analysis of the tight junction protein ZO-1 in colon sections from AOM/DSS-treated mice. Representative images of the percentage of Z0-1-positive cells (black arrows) in the colonic mucosa. Magnification, $\times 400$. Scale bar, $50 \mu \mathrm{m}$.

Z0-1, zonula occludens 1; OCLN, occludin; CLDN4, claudin 4; GAPDH, glyceraldehyde-3-phosphate dehydrogenase; M_Con., male control mice; M_AOM/DSS, male mice treated with AOM/DSS; M_AOM/DSS/E2, AOM/DSS-treated male mice administered estradiol; F_Con., female control mice; F_AOM/DSS, female mice treated with AOM/DSS. * $\mathrm{p}<0.05$ for comparison between two groups.

dependent pro-inflammatory cytokines, enzymes, and genes. At first, we determined the expression level of Krüppel-like factor 4 (KLF4) gene which is reported as an important mediator of DSSinduced colonic inflammation by modulating $N F-\kappa B$ signaling pathway. ${ }^{34}$ The AOM/DSS-treated male mice with E2 administration showed significantly reduced level of KLF4 mRNA expression in comparison to the AOM/DSS-treated male mice $(\mathrm{p}=0.018)$ (Fig. 4A). The AOM/DSS-treated female mice showed reduced level of KLF4 mRNA expression compare to the AOM/DSS-treated male mice without statistical significance (Fig. 4A). Consistent with KLF4 gene expression, NF- $\mathrm{KB}$ expression in the nucleus was decreased in the AOM/DSS-treated male mice with E2 administration compared to the AOM/DSS-treated male mice (Fig. 4B). The AOM/DSS-treated female mice also reduced NF- $\kappa \mathrm{B}$ expres- sion compared to the AOM/DSS-treated male mice (Fig. 4B).

Next, we assessed the levels of two major pro-inflammatory enzymes (i.e., inducible nitric oxide synthase [iNOS] and cyclooxygenase- 2 [COX-2]) which are mainly regulated by NF- $\mathrm{B}$ by Western blot analysis. The AOM/DSS-treated male mice with E2 administration showed significantly reduced levels of iNOS and COX-2 expression compared to the AOM/DSS-treated male mice ( $p=0.004$ for iNOS and $p=0.003$ for COX-2) (Fig. 4C). The AOM/ DSS-treated female mice showed reduced level of pro-inflammatory enzymes compare to the AOM/DSS-treated male mice without statistical significance (Fig. 4C). Subsequently, we measured mRNA expression of NF- $\kappa \mathrm{B}$-related pro-inflammatory cytokines (i.e., TNF- $\alpha$, IL-6, and IL-1 $\beta$ ) in the intestinal mucosa by qRTPCR. The expression level of TNF- $\alpha$, IL-6, and IL- $1 \beta$ in the AOM/ 

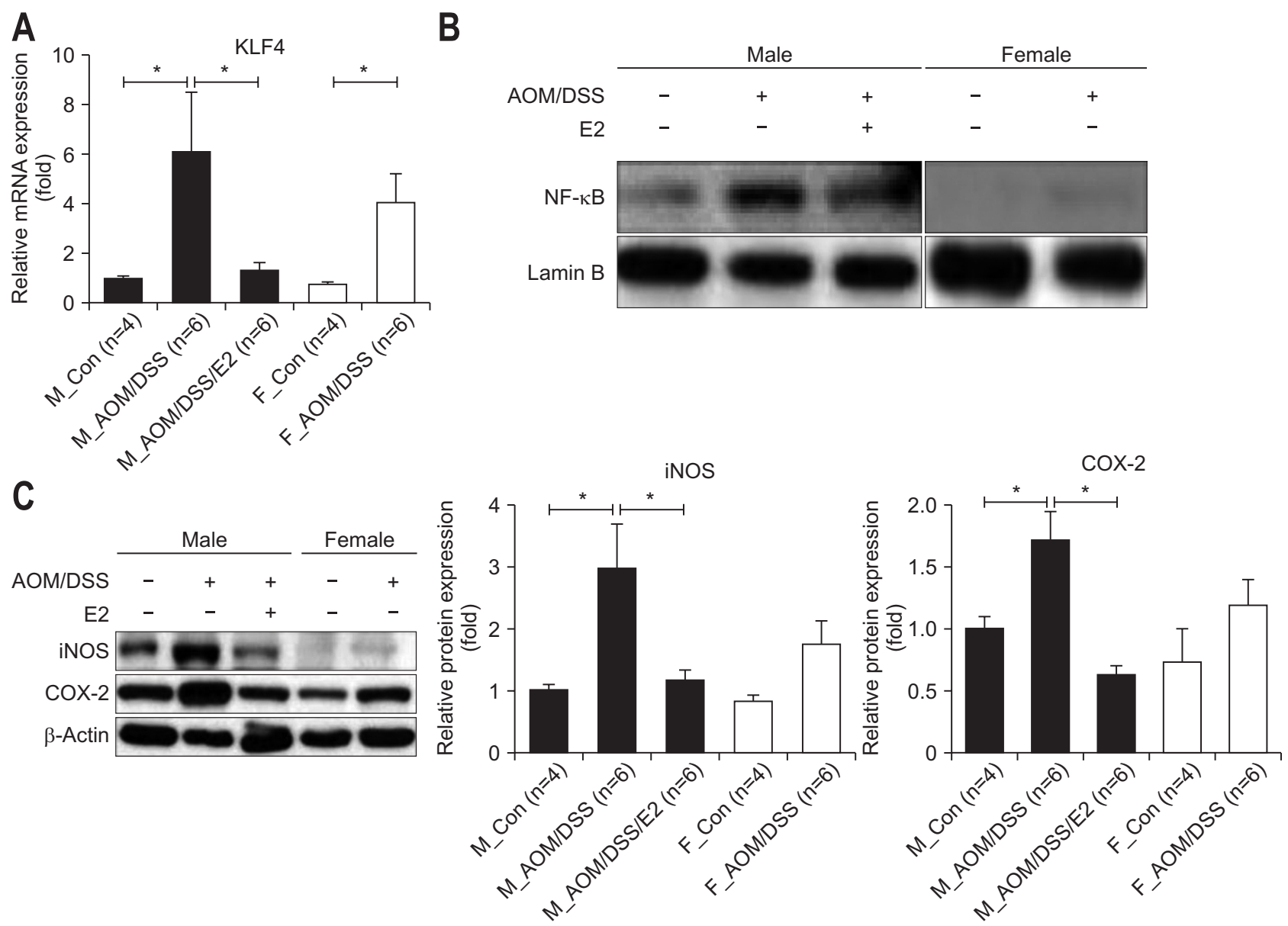

Fig. 4. Inhibitory effects of $17 \beta$-estradiol (E2) on the expression of nuclear factor $\kappa \mathrm{B}(\mathrm{NF}-\kappa \mathrm{B})$-dependent inflammatory mediators in azoxymethane/dextran sulfate sodium (AOM/DSS)-induced colitis in mice. (A) AOM/DSS-induced Krüppel-like factor 4 (KLF4) mRNA expression was inhibited by E2. Expression of KLF4 in the colonic mucosa was measured by qRT-PCR analysis. (B) AOM/DSS-induced NF- $\mathrm{B}$ activation was reduced by E2. Expression of NF- $\mathrm{B}$ in the nuclear fraction within the colonic mucosa was assessed by western blot analysis. (C) AOM/DSS-induced inducible nitric oxide synthase (iNOS) and cyclooxygenase-2 (COX-2) protein expression was diminished by E2. Expression of iNOS and COX-2 was measured by Western blot analysis. (D-F) Expression of tumor necrosis factor- $\alpha$ (TNF- $\alpha$ ), interleukin (IL)-6, and IL- $1 \beta$ in the colonic mucosa was detected by qRT-PCR analysis. (G, H) Expression of estrogen receptor (ER) $\alpha$ and ER $\beta$ in the colonic mucosa was detected by qRT-PCR analysis. GAPDH, Lamin B, and $\beta$-actin expression were used as internal controls to normalize expression.

pRT-PCR, quantitative real-time polymerase chain reaction; GAPDH, glyceraldehyde-3-phosphate dehydrogenase; M_Con., male control mice; M_ AOM/DSS, male mice treated with AOM/DSS; M_AOM/DSS/E2, AOM/DSS-treated male mice administered estradiol; F_Con., female control mice; F_AOM/DSS, female mice treated with AOM/DSS. *p $<0.05$ for comparison between two groups.

DSS-treated male mice with E2 administration decreased compared to the AOM/DSS-treated male mice ( $\mathrm{p}=0.002$ for TNF- $\alpha$, $\mathrm{p}=0.012$ for $\mathrm{IL}-6$, and $\mathrm{p}<0.001$ for $\mathrm{IL}-1 \beta$ ) (Fig. $4 \mathrm{D}-\mathrm{F}$ ). Moreover, the AOM/DSS-treated female mice enhanced pro-inflammatory cytokines compared to the AOM/DSS-treated male mice ( $\mathrm{p}=0.024$ for IL-1 $\beta$ ) (Fig. 4D-F). The levels of pro-inflammatory enzyme and cytokine gene expression were consistent with the results of microscopic damage index in male group. Taken together, these observations suggested that E2 has anti-inflammatory effects, especially suppressing $\mathrm{NF}-\kappa \mathrm{B}-$ related inflammatory mediators.

\section{The expressions of ER $\alpha$ and ER $\beta$ were differently regu- lated by $\mathrm{E} 2$ supply in colonic mucosa}

Furthermore, we measured mRNA expression of ERs in the intestinal mucosa by qRT-PCR to verify the role of ERs on in- flammation-mediated colonic mucosal permeability. As shown in Fig. 4D, ER $\alpha$ expression was significantly increased by AOM/ DSS-treatment on both male and female group compared to control mice ( $p=0.093$ for M_Con. vs M_AOM/DSS, $p=0.026$ for F_Con. vs F_AOM/DSS) (Fig. 4G). And the level was decreased by E2 administration compared to M_AOM/DSS group, but not significantly. On the contrary, ER $\beta$ expression was strongly repressed by AOM/DSS-treatment on both sex compared to control mice ( $p<0.001$ for M_Con. vs M_AOM/DSS, $p<0.001$ for F_Con. vs F_AOM/DSS) (Fig. 4H). And the decreased level was significantly recovered by E2 supply compared to M_AOM/DSS $(\mathrm{p}<0.001)$ (Fig. 4H). 

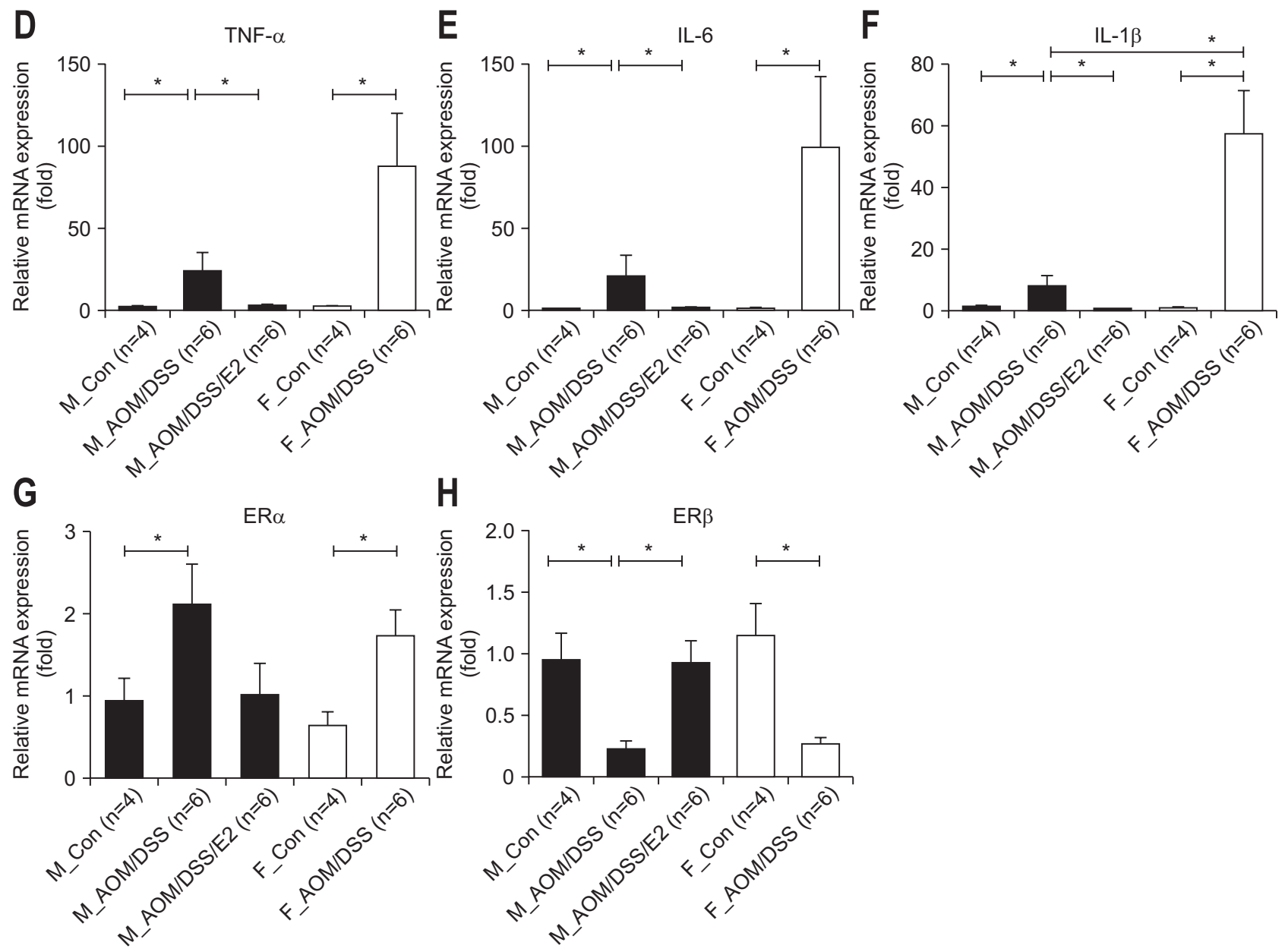

Fig. 4. Continued.

\section{DISCUSSION}

In the present study, we found that E2 has protective role on intestinal barrier in the transition stage from inflammation to cancer in colon. To investigate the estrogen effects on that, we used AOM/DSS-treated colitis mouse model, and collected and assessed samples of colon mucosa at week 4 after AOM injection in ICR mice. According to in vivo study of AOM/DSSinduced colitis and CRC mice model, inflammation score was the highest at week 2 without colonic neoplasm development and multiplicity of colonic neoplasm was increased in a timedependent manner, ${ }^{7}$ which had been confirmed by our previous data. $^{28}$ Suzuki et al. ${ }^{7}$ suggested that week 4 after AOM injection is the transition stage to move from inflammation to cancer. As TJ is very important for the homeostasis of organism we hypnotized that damage of $\mathrm{TJ}$ by acute inflammation contributes to cancer development of AOM/DSS-induced CRC mice model. In addition, as E2 is known to maintain the mucosal barrier function $^{35}$ we had interest in sex difference. Our team showed that AOM/DSS-treated female mice have significantly lower tumor multiplicity and incidence compared to AOM/DSS-treated male mice. ${ }^{30}$ Regarding the underlying anti-inflammation and anticancer mechanism of estrogen in in vivo and in vitro systems our data suggested that estrogen has pivotal role on $\mathrm{NF}-\kappa \mathrm{B}-$ dependent signaling pathway and anti-oxidant enzyme gene expressions in inflammation or carcinogenesis in in vivo ${ }^{28}$ and in vitro ${ }^{36}$ systems.

Mucins are the main components of mucus that act the first protective barrier for epithelial surface. Mucins can be classified into two types, namely secreted mucins and membrane bound (transmembrane) mucins based on the structure and localization. Secreted mucins form a protective layer over organs that contact the external environment and creates a physical barrier against pathogens. Among secreted gel forming mucin, MUC2 is the major intestinal mucin, expressed by goblet cells of the small intestine $e^{37-39}$ and colorectum. ${ }^{34,37}$ MUC2 knockout mice exhibited colonic inflammation and spontaneous development of colitis and $\mathrm{CRC}{ }^{40}$ and MUC2 deficiency alters inflammatory and metabolic pathways in the mouse intestinal mucosa. ${ }^{41}$ In addition, Hsu et al. ${ }^{42}$ demonstrated MUC2 suppressed the migration of colon cancer cells in vitro and dramatically diminished liver metastasis in vivo through IL-6 signaling. In concordance 
with these studies, our data also showed that mRNA expression of MUC2 was decreased in AOM/DSS-treated male mice compared to control male mice. The diminished expression of MUC2 was significantly recovered by E2 supplementation in AOM/ DSS-treated male mice. Membrane bound mucins have role in various signaling pathways by locating in plasma membrane. Alteration of mucin gene expression or glycosylation has been seen in various types of pathological conditions such as cancers and IBD, which highlight the importance of mucin in maintaining homeostasis. ${ }^{43}$ MUC4 is one of membrane-bound mucins, expressed by goblet and absorptive cells of the small intestine and colon. ${ }^{44}$ According to Das et al., ${ }^{22}$ MUC4 knockout mice displayed increased resistance to DSS-induced colitis by showing reduced tumor burden. Also, neutrophil elastase, a major inflammatory protease released by infiltrating neutrophils, upregulated MUC4 gene expression in normal human bronchial epithelial cells. ${ }^{45}$ Our data also showed that the mRNA expression of MUC4 was strongly enhanced in AOM/DSS-treated male mice compared to control male mice. The enhanced expression of MUC4 was significantly inhibited by E2 supplementation in AOM/DSS-treated male mice. However, there was no difference between male and female in MUC2 and MUC4 mRNA expression. Taken together, the present study supports that E2 enhanced intestinal barrier function by modulating mucin gene expression in colon mucus.

TJs are multi-protein complexes that form a barrier which is selectively permeable seal between adjacent epithelial cells and demarcates the boundary between apical and basolateral membrane domains. ${ }^{23}$ Major components of TJs are ZOs, OCLN, CLDNs, and junctional adhesion molecules (JAM). The modification of TJ molecules and paracellular permeability on the barrier function are regulated by various extracellular stimuli and are closely associated with maintaining homeostasis. ${ }^{46}$ In ulcerative colitis patients, over expressions of TJ molecules such as OCLN, CLDN-1, -4, and JAM-A, and down regulation of CLDN-2 expression were observed. ${ }^{47}$ According to Ueda et al., ${ }^{48}$ disruption of CLDN-4-mediated TJ molecules enhanced cancer cell invasion and metastasis in human CRC, and also CLDN-4 knockdown using siRNA in SW480 CLDN-4-positive CRC cells upregulated cell motility, whereas no significant change was detected in cell proliferation. In male gastroesophageal reflux disease (GERD) patients, inhibited mRNA expression of OCLN and enhanced mRNA expression of CLDN-1, CLDN-4, and NK1R except ZO-1 were observed. Furthermore, the research group investigated that female GERD patients were affected more by reflux symptoms than males. ${ }^{49}$ In the present study, we found that exogenous estrogen has protective role on the colitis-mediated intestinal permeability through up-regulation of TJ molecules such as ZO-1, OCLN, and CLDN4. Furthermore, TJ molecules mRNA expressions and Z0-1-positive cells stained into the plasma membrane were differently regulated by sex in AOM/ DSS-treated mice model. These results suggest that exogenous and endogenous estrogen would somehow differently regulate the TJ molecule expression. We need further investigation to figure out the regulatory mechanism of estrogen on cellular permeability and localization and expression of TJ molecules.

The NF- $\kappa \mathrm{B}$ proteins are normally sequestered in the cytoplasm of unstimulated cells via interactions with a family of inhibitor proteins called $\mathrm{I}_{\kappa} \mathrm{Bs} .{ }^{50}$ A well-known function of NF- $\kappa \mathrm{B}$ which is a family of inducible transcription factors is regulation of a number of target genes involved in different processes of the immune and inflammatory responses. ${ }^{51}$ Recently, deregulated NF- $\mathrm{B}$ activation has been implicated in the pathogenesis of a number of inflammatory diseases, such as rheumatoid arthritis, IBD, multiple sclerosis, atherosclerosis, systemic lupus erythematosus, type I diabetes, chronic obstructive pulmonary disease and asthma. ${ }^{52}$ Especially, strong evidence suggests the involvement of NF- $\kappa \mathrm{B}$ in the pathogenesis of $\mathrm{IBD}^{53}$ and colitisassociated $\mathrm{CRC}^{54}$ Constitutive NF- $\mathrm{KB}$ activation ${ }^{55,56}$ and genetic mutations in NFKB1 gene ${ }^{57}$ and NF- $\kappa \mathrm{B}$ target genes, ${ }^{58}$ such as TNF- $\alpha$, IL-1, and IL-6, have been associated with human IBD and/or CRC. Furthermore, E2 treatment ameliorates gastric ulcer index, colonic damage score, and the severity of ulcers and colitis in acetic acid-induced gastric and colonic injuries in rats. ${ }^{59}$ In concordance with these studies, our study indicates that E2 shows inhibitory effects on the $\mathrm{NF}-\kappa \mathrm{B}$-dependent signaling pathway through directly inhibits KLF4 mRNA expression and $\mathrm{NF}-\kappa \mathrm{B}$ activation, and negatively regulates mRNA and/or protein expression of pro-inflammatory mediators such as iNOS, COX-2, TNF- $\alpha$, IL-6, and IL- $1 \beta$ which are regulated by NF- $\kappa$ Bdependent signaling axis. Finally, E2 suppressed the inflammatory cell infiltration and microscopic damage score used as inflammatory index in AOM/DSS-treated male mice.

Numerous evidence has been reported in the literature ascribing anti-inflammatory and anti-tumorigenic effects of estrogens in the colon. ${ }^{60,61}$ Furthermore, the risk of colon cancer in postmenopausal women was suppressed by hormone replacement therapy. ${ }^{62}$ Generally, intracellular signaling of estrogens is mediated by its association with their receptors, ER $\alpha$ and ER $\beta$. In colonic epithelium, ER $\beta$ is the predominant functional ER subtype expressed ${ }^{63}$ and its levels are inhibited in CRC compared to normal colonic mucosa. ${ }^{27}$ These findings suggest that protective role of estrogens in the colon may be exerted via ER $\beta$ signaling. Furthermore, Saleiro et al. ${ }^{64}$ reported that ER $\beta$ KO mice showed increased expression of inflammation-related molecules and MUC1 and decreased expression of MUC2. Consistent with these studies, our results show that suppressed expression of ER $\beta$ by AOM/DSS treatment was recovered by E2 supply, in contrary to ER $\alpha$ expression.

In conclusion, E2 administration suppressed the colitisinduced intestinal permeability and inflammation in experimentally induced colitis male mice. The protective effects of E2 appear to be associated with increment the mRNA expression level of MUC2 and TJ molecules, and blockade of pro-inflammatory 
mediators modulated by NF- $\mathrm{BB}$-dependent signaling pathway. Especially, the gene expressions of TJ molecules were differently regulated by sex in AOM/DSS-induced colitis. These findings may provide a new mechanistic explanation by which E2 regulates the intestinal permeability and inflammation during the transition stage from colitis to CRC through regulation of ERs expression.

\section{CONFLICTS OF INTEREST}

No potential conflict of interest relevant to this article was reported.

\section{ACKNOWLEDGEMENTS}

This work was supported by a grant from the National Research Foundation of Korea (NRF) funded by the government of the Republic of Korea (2016R1A2B4013133).

Author Contribution: Song $\mathrm{CH}$ drafted manuscript and performed molecular experiments; Kim $\mathrm{N}$ designed and supervised the experiments, and revised manuscript, Sohn SH, Lee SM, and Nam RH performed animal experiments; Na HY advised the IHC experiment of ZO-1; Lee DH and Surh YJ revised manuscript.

\section{REFERENCES}

1. Siegel RL, Miller KD, Jemal A. Cancer statistics, 2018. CA Cancer J Clin 2018;68:7-30.

2. Jung KW, Won YJ, Oh CM, et al. Cancer statistics in Korea: incidence, mortality, survival, and prevalence in 2014. Cancer Res Treat 2017;49:292-305.

3. Danese S, Malesci A, Vetrano S. Colitis-associated cancer: the dark side of inflammatory bowel disease. Gut 2011;60:1609-1610.

4. Popivanova BK, Kitamura K, Wu Y, et al. Blocking TNF-alpha in mice reduces colorectal carcinogenesis associated with chronic colitis. J Clin Invest 2008;118:560-570.

5. Grivennikov S, Karin E, Terzic J, et al. IL-6 and Stat3 are required for survival of intestinal epithelial cells and development of colitisassociated cancer. Cancer Cell 2009;15:103-113.

6. Tanaka T, Kohno H, Suzuki R, Yamada Y, Sugie S, Mori H. A novel inflammation-related mouse colon carcinogenesis model induced by azoxymethane and dextran sodium sulfate. Cancer Sci 2003;94:965-973.

7. Suzuki R, Kohno H, Sugie S, Tanaka T. Sequential observations on the occurrence of preneoplastic and neoplastic lesions in mouse colon treated with azoxymethane and dextran sodium sulfate. Cancer Sci 2004;95:721-727.

8. Thaker AI, Shaker A, Rao MS, Ciorba MA. Modeling colitisassociated cancer with azoxymethane (AOM) and dextran sulfate sodium (DSS). J Vis Exp 2012;(67):4100.

9. Tardieu D, Jaeg JP, Cadet J, Embvani E, Corpet DE, Petit C. Dextran sulfate enhances the level of an oxidative DNA damage biomarker, 8-oxo-7,8-dihydro-2'-deoxyguanosine, in rat colonic mucosa. Cancer Lett 1998;134:1-5.

10. De Robertis M, Massi E, Poeta ML, et al. The AOM/DSS murine model for the study of colon carcinogenesis: from pathways to diagnosis and therapy studies. J Carcinog 2011;10:9.

11. Suzuki R, Kohno H, Sugie S, Nakagama H, Tanaka T. Strain differences in the susceptibility to azoxymethane and dextran sodium sulfate-induced colon carcinogenesis in mice. Carcinogenesis 2006;27:162-169.

12. Shan M, Gentile M, Yeiser JR, et al. Mucus enhances gut homeostasis and oral tolerance by delivering immunoregulatory signals. Science 2013;342:447-453.

13. Johansson ME. Mucus layers in inflammatory bowel disease. Inflamm Bowel Dis 2014;20:2124-2131.

14. Fishman JE, Levy G, Alli V, Zheng X, Mole DJ, Deitch EA. The intestinal mucus layer is a critical component of the gut barrier that is damaged during acute pancreatitis. Shock 2014;42:264-270.

15. Krug SM, Schulzke JD, Fromm M. Tight junction, selective permeability, and related diseases. Semin Cell Dev Biol 2014;36:166176.

16. Wehkamp J, Koslowski M, Wang G, Stange EF. Barrier dysfunction due to distinct defensin deficiencies in small intestinal and colonic Crohn's disease. Mucosal Immunol 2008;1 Suppl 1:S67S74.

17. Wrzosek L, Miquel S, Noordine ML, et al. Bacteroides thetaiotaomicron and Faecalibacterium prausnitzii influence the production of mucus glycans and the development of goblet cells in the colonic epithelium of a gnotobiotic model rodent. BMC Biol 2013; 11:61.

18. Ulluwishewa D, Anderson RC, McNabb WC, Moughan PJ, Wells JM, Roy NC. Regulation of tight junction permeability by intestinal bacteria and dietary components. J Nutr 2011;141:769-776.

19. Groschwitz KR, Hogan SP. Intestinal barrier function: molecular regulation and disease pathogenesis. J Allergy Clin Immunol 2009;124:3-20.

20. Velcich A, Yang W, Heyer J, et al. Colorectal cancer in mice genetically deficient in the mucin Muc2. Science 2002;295:17261729.

21. Yang K, Popova NV, Yang WC, et al. Interaction of Muc2 and Apc on Wnt signaling and in intestinal tumorigenesis: potential role of chronic inflammation. Cancer Res 2008;68:7313-7322.

22. Das S, Rachagani S, Sheinin Y, et al. Mice deficient in Muc4 are resistant to experimental colitis and colitis-associated colorectal cancer. Oncogene 2016;35:2645-2654.

23. Lee SH. Intestinal permeability regulation by tight junction: implication on inflammatory bowel diseases. Intest Res 2015;13:11-18.

24. González-Mariscal L, Lechuga S, Garay E. Role of tight junctions in cell proliferation and cancer. Prog Histochem Cytochem 2007; 42:1-57.

25. Lee YJ, Hussain Z, Huh CW, Lee YJ, Park H. Inflammation, impaired motility, and permeability in a guinea pig model of postoperative ileus. J Neurogastroenterol Motil 2018;24:147-158. 
26. Kim JJ, Kim N, Choi YJ, Kim JS, Jung HC. Increased TRPV1 and PAR2 mRNA expression levels are associated only with the esophageal reflux symptoms, but not with the extraesophageal reflux symptoms. Medicine (Baltimore) 2016;95:e4387.

27. Konstantinopoulos PA, Kominea A, Vandoros G, et al. Oestrogen receptor beta (ERbeta) is abundantly expressed in normal colonic mucosa, but declines in colon adenocarcinoma paralleling the tumour's dedifferentiation. Eur J Cancer 2003;39:1251-1258.

28. Son HJ, Sohn SH, Kim N, et al. Effect of estradiol in an azoxymethane/dextran sulfate sodium-treated mouse model of colorectal cancer: implication for sex difference in colorectal cancer development. Cancer Res Treat. Epub 2018 Aug 1. https://doi. org/10.4143/crt.2018.060.

29. Harris HA. Estrogen receptor-beta: recent lessons from in vivo studies. Mol Endocrinol. 2007;21:1-13.

30. Lee SM, Kim N, Son HJ, et al. The effect of sex on the azoxymethane/dextran sulfate sodium-treated mice model of colon cancer. J Cancer Prev 2016;21:271-278.

31. Yum HW, Zhong X, Park J, et al. Oligonol inhibits dextran sulfate sodium-induced colitis and colonic adenoma formation in mice. Antioxid Redox Signal 2013;19:102-114.

32. Park YH, Kim N, Shim YK, et al. Adequate dextran sodium sulfateinduced colitis model in mice and effective outcome measurement method. J Cancer Prev 2015;20:260-267.

33. Kim YS, Ho SB. Intestinal goblet cells and mucins in health and disease: recent insights and progress. Curr Gastroenterol Rep 2010; 12:319-330.

34. Ghaleb AM, Laroui H, Merlin D, Yang VW. Genetic deletion of Klf4 in the mouse intestinal epithelium ameliorates dextran sodium sulfate-induced colitis by modulating the NF-kappaB pathway inflammatory response. Inflamm Bowel Dis 2014;20:811-820.

35. Grishina I, Fenton A, Sankaran-Walters S. Gender differences, aging and hormonal status in mucosal injury and repair. Aging Dis 2014;5:160-169.

36. Son HJ, Kim N, Song CH, Lee SM, Lee HN, Surh YJ. 17 $\beta$-Estradiol reduces inflammation and modulates antioxidant enzymes in colonic epithelial cells. Korean J Intern Med. Epub 2018 Oct 22. https://doi.org/10.3904/kjim.2018.098.

37. Audie JP, Janin A, Porchet N, Copin MC, Gosselin B, Aubert JP. Expression of human mucin genes in respiratory, digestive, and reproductive tracts ascertained by in situ hybridization. J Histochem Cytochem 1993;41:1479-1485.

38. Buisine MP, Devisme L, Copin MC, et al. Developmental mucin gene expression in the human respiratory tract. Am J Respir Cell Mol Biol 1999;20:209-218.

39. Gum JR Jr, Hicks JW, Toribara NW, Siddiki B, Kim YS. Molecular cloning of human intestinal mucin (MUC2) cDNA: identification of the amino terminus and overall sequence similarity to preprovon Willebrand factor. J Biol Chem 1994;269:2440-2446.

40. Van der Sluis M, De Koning BA, De Bruijn AC, et al. Muc2-deficient mice spontaneously develop colitis, indicating that MUC2 is critical for colonic protection. Gastroenterology 2006;131:117-
129.

41. Tadesse S, Corner G, Dhima E, et al. MUC2 mucin deficiency alters inflammatory and metabolic pathways in the mouse intestinal mucosa. Oncotarget 2017;8:71456-71470.

42. Hsu HP, Lai MD, Lee JC, et al. Mucin 2 silencing promotes colon cancer metastasis through interleukin-6 signaling. Sci Rep 2017;7: 5823.

43. Dhanisha SS, Guruvayoorappan C, Drishya S, Abeesh P. Mucins: structural diversity, biosynthesis, its role in pathogenesis and as possible therapeutic targets. Crit Rev Oncol Hematol 2018;122:98122.

44. Andrianifahanana M, Moniaux N, Batra SK. Regulation of mucin expression: mechanistic aspects and implications for cancer and inflammatory diseases. Biochim Biophys Acta 2006;1765:189222

45. Fischer BM, Cuellar JG, Diehl ML, et al. Neutrophil elastase increases MUC4 expression in normal human bronchial epithelial cells. Am J Physiol Lung Cell Mol Physiol 2003;284:L671-L679.

46. Nusrat A, Turner JR, Madara JL. Molecular physiology and pathophysiology of tight junctions. IV. Regulation of tight junctions by extracellular stimuli: nutrients, cytokines, and immune cells. Am J Physiol Gastrointest Liver Physiol 2000;279:G851-G857.

47. Vetrano S, Rescigno M, Cera MR, et al. Unique role of junctional adhesion molecule-A in maintaining mucosal homeostasis in inflammatory bowel disease. Gastroenterology 2008;135:173-184.

48. Ueda J, Semba S, Chiba H, et al. Heterogeneous expression of claudin-4 in human colorectal cancer: decreased claudin-4 expression at the invasive front correlates cancer invasion and metastasis. Pathobiology 2007;74:32-41.

49. Kim JJ, Kim N, Park JH, et al. Comparison of tight junction protein-related gene mRNA expression levels between male and female gastroesophageal reflux disease patients. Gut Liver 2018;12: 411-419.

50. Sun SC. Non-canonical NF-kappaB signaling pathway. Cell Res 2011;21:71-85.

51. Oeckinghaus A, Ghosh S. The NF-kappaB family of transcription factors and its regulation. Cold Spring Harb Perspect Biol 2009;1: a000034.

52. Pai S, Thomas R. Immune deficiency or hyperactivity-NF-kappaB illuminates autoimmunity. J Autoimmun 2008;31:245-251.

53. Liu T, Zhang L, Joo D, Sun SC. NF-kappaB signaling in inflammation. Signal Transduct Target Ther 2017;2:17023.

54. Viennois E, Chen F, Merlin D. NF-kappaB pathway in colitisassociated cancers. Transl Gastrointest Cancer 2013;2:21-29.

55. Schreiber S, Nikolaus S, Hampe J. Activation of nuclear factor kappa B inflammatory bowel disease. Gut 1998;42:477-484.

56. Greten FR, Eckmann L, Greten TF, et al. IKKbeta links inflammation and tumorigenesis in a mouse model of colitis-associated cancer. Cell 2004;118:285-296.

57. Karban AS, Okazaki T, Panhuysen CI, et al. Functional annotation of a novel NFkB1 promoter polymorphism that increases risk for ulcerative colitis. Hum Mol Genet 2004;13:35-45. 
58. Atreya I, Atreya R, Neurath MF. NF-kappaB in inflammatory bowel disease. J Intern Med 2008;263:591-596.

59. Günal 0, Oktar BK, Ozçinar E, Sungur M, Arbak S, Yeğen B. Estradiol treatment ameliorates acetic acid-induced gastric and colonic injuries in rats. Inflammation 2003;27:351-359.

60. Harnish DC, Albert LM, Leathurby Y, et al. Beneficial effects of estrogen treatment in the HLA-B27 transgenic rat model of inflammatory bowel disease. Am J Physiol Gastrointest Liver Physiol 2004;286:G118-G125.

61. Kennelly R, Kavanagh DO, Hogan AM, Winter DC. Oestrogen and the colon: potential mechanisms for cancer prevention. Lancet
Oncol 2008;9:385-391.

62. Grodstein F, Newcomb PA, Stampfer MJ. Postmenopausal hormone therapy and the risk of colorectal cancer: a review and meta-analysis. Am J Med 1999;106:574-582.

63. Campbell-Thompson M, Lynch IJ, Bhardwaj B. Expression of estrogen receptor (ER) subtypes and ERbeta isoforms in colon cancer. Cancer Res 2001;61:632-640.

64. Saleiro D, Murillo G, Benya RV, Bissonnette M, Hart J, Mehta RG. Estrogen receptor-beta protects against colitis-associated neoplasia in mice. Int J Cancer 2012;131:2553-2561. 